\title{
The effect of vaginal bleeding and non-spesific pelvic pain on pregnancy outcomes in subchorionic hematomas cases
}

\author{
Talip Karaçor ${ }^{1}$, Mehmet Bülbüll ${ }^{1}$, Mehmet Can Nacar ${ }^{1}$, Pınar Kırıcı ${ }^{1}$, \\ Nurullah Peker², Elif Ağaçayak² \\ ${ }^{1}$ Adiyaman University Faculty of Medicine, Department of Obstetrics and Gynecology, Adiyaman, Turkey \\ ${ }^{2}$ Depertment of Gynecology and Obstetrics, Faculty of Medicine, Dicle University, Diyarbakır, Turkey
}

\begin{abstract}
Objectives: To determine the clinical differences and factors affecting early pregnancy outcome in the first and early second trimester subchorionic hematoma cases.

Material and methods: This study involved with the retrospective analysis and evaluation of 81 cases diagnosed with subchorionic hematoma. The patients were grouped according to the gestational periods, symptoms at the time of admission, ratio of surrounding hematoma to the gestational sac, and whether there was a pregnancy loss. The groups were compared according to the clinical features and pregnancy outcomes.

Results: The ratio of surrounding hematoma to the gestational sac in the group with pregnancy loss was significantly higher $(p=0.002)$. When the cut-off value was $35.5 \%$, it could determine the possibility of a complication in pregnancy with $70 \%$ sensitivity and $75 \%$ specificity. Nonspecific pelvic pain were significantly higher in the pregnancy loss group than in the other group. Logistic regression analysis was performed to determine the effect of these two parameters on the pregnancy outcome. Although the presence of non-specific pelvic pain is more in the group with pregnancy loss; there was no effect of on pregnancy outcome $(p=0.141)$. The risk of pregnancy loss increased 4.5 fold if the ratio of ScH to gestational sac was above $35 \%(p=0.027)$.

Conclusions: In the cases of subchorionic hematoma, we concluded that when the ratio of surrounding hematoma to the gestational sac increased and when it was accompanied by nonspecific pelvic pain, the hospitalization period of the patients increased and the ratio of pregnancy loss was higher.
\end{abstract}

Key words: subchorionic hematoma; vaginal bleeding; pelvic pain; first trimester; complication

Ginekologia Polska 2019; 90, 11: 656-661

\section{INTRODUCTION}

Subchorionic hematoma $(\mathrm{ScH})$ is defined as a crescent-shaped, echo-free area between the chorionic membrane and the myometrium. The incidence ranges from $1.3 \%$ to $3.1 \%$ [1]. It is a rare but serious cause of vaginal bleeding that is extremely common in the early gestational weeks. Although its etiology is not known exactly, the increase of assisted reproductive techniques and low-molecular-weight heparin and aspirin use are among the risk factors [2]. It is usually diagnosed in patients admitted due to vaginal bleeding. In addition, non-specific pelvic pain (NsPP), such as low back, groin and around of umbilicus pains may accompany vaginal bleeding. $\mathrm{ScH}$ can be diagnosed in routine ultrasonography examinations for asymptomatic patients [3-5].
It is generally classified as small, medium, or large according to the ratio of the diameter of the hematoma to that of the gestational sac [6]. In addition, $\mathrm{ScH}$ is also classified according to its surrounding ratio to the gestational sac [7]. In cases with $\mathrm{ScH}$, early pregnancy complications like missed abortion and spontaneous complete or incomplete abortion and late complications of pregnancy, such as premature rupture of membranes, preterm delivery, and intrauterine growth retardation, have been reported to be more frequent [8-10].

In almost all of the previous studies, the effect of hematoma size on early and late pregnancy complications in patients with first trimester period $\mathrm{ScH}$ was evaluated. The study evaluating the effect of NsPP on early complications of $\mathrm{ScH}$ cases is not available in the literature. 
The aim of this study was to determine the parameters that affect the early pregnancy losses except hematoma size in the first and early second trimester $\mathrm{ScH}$ cases. In our current study, we found that pregnancy was more complicated in $\mathrm{ScH}$ cases with non-specific pelvic pain.

\section{MATERIAL AND METHODS}

The study was approved by the "University Local Ethics Committee" before a retrospective file search was conducted for the study. The archive files, ultrasonography reports, and image records of 684 patients hospitalized with vaginal bleeding in "University Obstetrics and Gynecology Department" between January 2015 and October 2018 were reviewed. We detected total of 106 patients who had ultrasonography reports and visual hematoma measurement records.

A total of $25 \mathrm{ScH}$ cases were excluded from the study which pregnancies without a fetal heartbeat on ultrasonography reports, multiple pregnancies, and pregnancies assisted by supportive reproduction methods. Because, almost all of the pregnancies formed by assisted reproductive technique had a history of using acetylsalicylic acid, low molecular heparin or depot progesterone.

In addition, other pregnant women who developed spontaneous pregnancy but who used acetylsalicylic acid (aspirin), low-molecular-weight heparin and warfarin for another indication were excluded from the study. Demographic data like age, gravida, parity, history of surgery, and pregnancy formation method were obtained from the patients' anamnesis information. In addition, the patients' initial complaints at the time of admission were determined and recorded. In our study, we classified the ScHs according to the surrounding ratio to the gestational sac. We compared the circumference of the entire gestational sac with the gestational sac surface separated from the myometrium, and thus we obtained the inclusion ratio of hematomas in the gestational sac. We indicated the results as a percentage. The ratio of surrounding hematoma to the gestational sac was determined using the Generic Area program on the GE Voluson P8 device. The measurement of subchorionic hematomas is shown in Figure 1. The measurements were evaluated by two obstetricians who were not aware of the study parameters. The other important parameter was NsPP. While evaluating the pain of low back, groin and around of umbilicus during pregnancy, all abdominal organs and musculoskeletal pathologies should be considered [11]. There was no organic pathology to explain the pain we identified as NsPP. Patients with musculoskeletal system, gastrointestinal and urinary tract pathologies were not evaluated as NsPP [12]. In our clinic, three questions are asked in the evaluation of the pain around the groin, waist and umbilicus in the pregnancy.

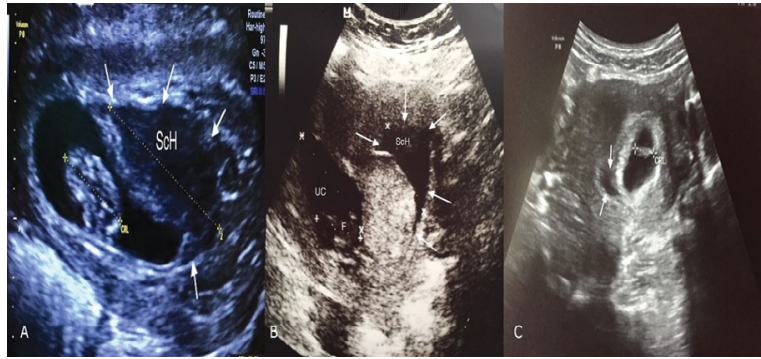

Figure 1. USG measurements of ScHs; A. A case of subchorionic hematoma (white arrow) surrounding approximately $50 \%$ of the gestational sac; B. A case of subchorionic hematoma (white arrow) that surrounds approximately $30 \%$ of the gestational sac; $\mathbf{C}$. A case of subchorionic hematoma (white arrow) that surrounds approximately $10 \%$ of the gestational sac; $\mathrm{ScH}-$ subchorionic hematoma; $\mathrm{CRL}$ — crown rump length (Fetus); UC — uterine cavity; F — fetus

Are these pains present during pregnancy and are they new?

Do you need analgesics in case of pain?

Do you wake up from your sleep in pain?

NsPP is added to the diagnosis of patients who give a yes response to the first question and either of the other two questions.

A gestational period of 42-98 days was classified as first trimester, while 99-140 days was classified as early second trimester pregnancy. Complications like missed abortion, complete or incomplete abortion, and termination of pregnancy after amnion fluid loss during the treatment before the $20^{\text {th }}$ gestastional week were evaluated within the early period pregnancy complication. These pregnancies resulted in loss. Pregnant women who reached over 20 weeks were accepted as the group without early period complications.

The patients were grouped as having vaginal bleeding and having vaginal bleeding + NsPP according to the symptoms at the time of admission. The patients were compared according to the gestational age, hematoma size, early complication status, and priority admission symptoms.

\section{Statistics}

The Statistical Package for the Social Sciences, version 15 (SPSS, Chicago, IL) program was used for the statistical analysis. The data were classified as being with or without normal distribution using the Kolmogorov-Smirnov test. Normally distributed data were evaluated using the independent sample t-test, while non-normally distributed data were compared using the Mann-Whitney U test. The Chi-square test was used for categorical variables. The level of statistical significance was set as $p<0.05$. Receiver operating characteristic (ROC) analysis was performed to determine the efficiency of the ratio of surrounding $\mathrm{ScH}$ to the gestational sac in foreseeing the gestational results. Logistic regression analysis was used to determine 
the risk factors affecting pregnancy outcome and to calculate odds ratio.

\section{RESULTS}

The number of pregnant women who were examined in the first trimester or early second trimester weeks in the University Obstetrics and Gynaecology outpatient clinic was 5.889 during the period included in the study. A total of 106 vaginal bleeding patients were detected. The incidence of vaginal bleeding in our clinic was $11.6 \%$. ScH was detected in 106 patients with vaginal bleeding. The incidence of $\mathrm{ScH}$ in patients with vaginal bleeding in our clinic was $15.4 \%$. The patients were treated with similar abortion imminence treatments during hospitalization. In our study, pregnancy loss was detected in 17 (21\%) patients. The other 64 (79\%) patients did not develop early pregnancy complications, and their pregnancies reached the 20th gestational week. The ratio of surrounding $\mathrm{ScH}$ to the gestational sac was statistically lower in the group without pregnancy loss than it was in the group with pregnancy loss $(p=0.002)$. The factors affecting pregnancy loss are listed in Table 1. First trimester (49-98 days) and early second trimester (99-140 days) pregnancies were compared in terms of the symptoms, hospitalization times, and ratio of surrounding hematoma to the gestational sac. In the first trimester group, 41 (83.7\%) patients had vaginal bleeding and $8(16.3 \%)$ had primary symptoms of vaginal bleeding + NsPP. In the early second trimester group, 17 (53.1\%) patients had vaginal bleeding and 15 (46.9\%) had primary symptoms of vaginal bleeding + NsPP.The difference between the two groups was statistically significant $(p=0.003)$. The hospitalization period in the first trimester group was statistically lower than that in the early second trimester group $(p<0.001)$. In the first trimester group, the ratio of surrounding $\mathrm{ScH}$ to the gestational sac was statistically lower than it was in the early second trimester group ( $p=0.005$; Tab. 2). The patients were compared according to the symptoms of vaginal bleeding and vaginal bleeding $+\mathrm{NsPP}$. In the vaginal bleeding + NsPP group, the ratio of surrounding hematoma to the gestational sac, duration of hospitalization, and pregnancy loss were statistically higher $(p=0.002, p<0.001, p<0.001$, $p<0.001, p<0.001$, respectively). There was no statistically significant effect of maternal age on symptoms in the $\mathrm{ScH}$ cases ( $p=0.623$; Tab. 3). ROC analysis was performed to determine the efficiency of the ratio of surrounding hematoma to the gestational sac in foreseeing gestational results. When the cutoff value was $35.5 \%$, it could determine the prognosis

Table 1. Clinical characteristics of patients according to pregnancy outcomes

\begin{tabular}{|c|c|c|c|c|}
\hline \multicolumn{2}{|l|}{ Variables } & No loss of pregnancies & Loss of Pregnancies & \multirow{2}{*}{$\mathbf{p}$} \\
\hline \multicolumn{2}{|l|}{$\mathbf{n}$} & 64 & 17 & \\
\hline \multicolumn{2}{|c|}{ Patients age [year], mean \pm SD } & $26.6 \pm 4.7$ & $28.2 \pm 4.5$ & 0.222 \\
\hline \multicolumn{2}{|c|}{ Gravity n (min-max) } & $3(1-5)$ & $4(1-7)$ & 0.212 \\
\hline \multicolumn{2}{|c|}{ Live Children n (min-max) } & $2(0-4)$ & $2(0-4)$ & 0.323 \\
\hline \multicolumn{2}{|c|}{ Abortion $n$ (min-max) } & $0(0-1)$ & $0(0-3)$ & 0.296 \\
\hline \multicolumn{2}{|c|}{ Pregnancy age [day], mean \pm SD } & $83.5 \pm 21.7$ & $96.1 \pm 28.3$ & 0.051 \\
\hline \multicolumn{2}{|c|}{ Hospitalization time [day], mean \pm SD } & $6.5 \pm 3.1$ & $7.9 \pm 4.9$ & 0.165 \\
\hline \multirow{2}{*}{$\begin{array}{l}\text { Symptoms } \\
\text { N (\%) }\end{array}$} & Bleeding & $51(79.7)$ & $7(41.2)$ & 0.002 \\
\hline & Bleeding + non-specific pelvic pain & $13(20.3)$ & $10(58.8)$ & \\
\hline \multicolumn{2}{|c|}{ GS Surrounded by Hematoma, $\%$ mean \pm SD } & $26.0 \pm 14.9$ & $39.3 \pm 15.5$ & 0.002 \\
\hline
\end{tabular}

SD — standard deviation; GS — gestational sac

\begin{tabular}{|c|c|c|c|c|}
\hline \multicolumn{2}{|l|}{ Variables } & First trimester & Early second trimester & \multirow{2}{*}{$\mathbf{p}$} \\
\hline \multicolumn{2}{|l|}{$\mathbf{n}$} & 49 & 32 & \\
\hline \multirow{2}{*}{$\begin{array}{l}\text { Symptom } \\
\mathrm{N}(\%)\end{array}$} & Bleeding & $41(83.7)$ & $17(53.1)$ & \multirow{2}{*}{0.003} \\
\hline & Bleeding \& non-specific pelvic pain & $8(16.3)$ & $15(46.9)$ & \\
\hline \multirow{2}{*}{$\begin{array}{l}\text { Outcome of pregnancy } \\
\mathrm{N}(\%)\end{array}$} & No loss of pregnancy & $41(83.7)$ & $23(71.9)$ & \multirow{2}{*}{0.202} \\
\hline & Loss of pregnancy & $8(16.3)$ & $9(28.1)$ & \\
\hline \multicolumn{2}{|c|}{ Hospitalization time (day), mean \pm SD } & $5.2 \pm 2.6$ & $9.3 \pm 3.4$ & $<0.001$ \\
\hline \multicolumn{2}{|c|}{ GS Surrounded by Hematoma, $\%$ (mean \pm SD) } & 24.915 .4 & $34.8 \pm 4.9$ & 0.005 \\
\hline
\end{tabular}

SD — standard deviation; GS — gestational sac 


\begin{tabular}{|l|l|l|l|}
\hline \multicolumn{3}{|c|}{ Table 3. Clinical features of the patients according to the symptoms } \\
\cline { 1 - 3 } Variables & Bleeding & $\begin{array}{l}\text { Bleeding + } \\
\text { non-specific } \\
\text { pelvic pain }\end{array}$ & p \\
\hline n & 58 & $\mathbf{2 3}$ & \\
\hline $\begin{array}{l}\text { GS Surrounded by } \\
\text { Hematoma, \% (mean } \pm \text { SD) }\end{array}$ & $22.9 \pm 13.1$ & $43.8 \pm 11.9$ & $<\mathbf{0 . 0 0 1}$ \\
\hline $\begin{array}{l}\text { Pregnancy Age (day), } \\
\text { (mean } \pm \text { SD) }\end{array}$ & $78.7 \pm 21.4$ & $105.0 \pm 17.9$ & $<\mathbf{0 . 0 0 1}$ \\
\hline $\begin{array}{l}\text { Patient Age (year) } \\
\text { (mean } \pm \text { SD) }\end{array}$ & $26.8 \pm 5.1$ & $27.3 \pm 3.8$ & 0.623 \\
\hline $\begin{array}{l}\text { Hospitalization time (day), } \\
\text { (mean } \pm \text { SD) }\end{array}$ & $5.8 \pm 2.7$ & $9.4 \pm 4.1$ & $<\mathbf{0 . 0 0 1}$ \\
\hline
\end{tabular}

SD — standard deviation; GS - gestational sac

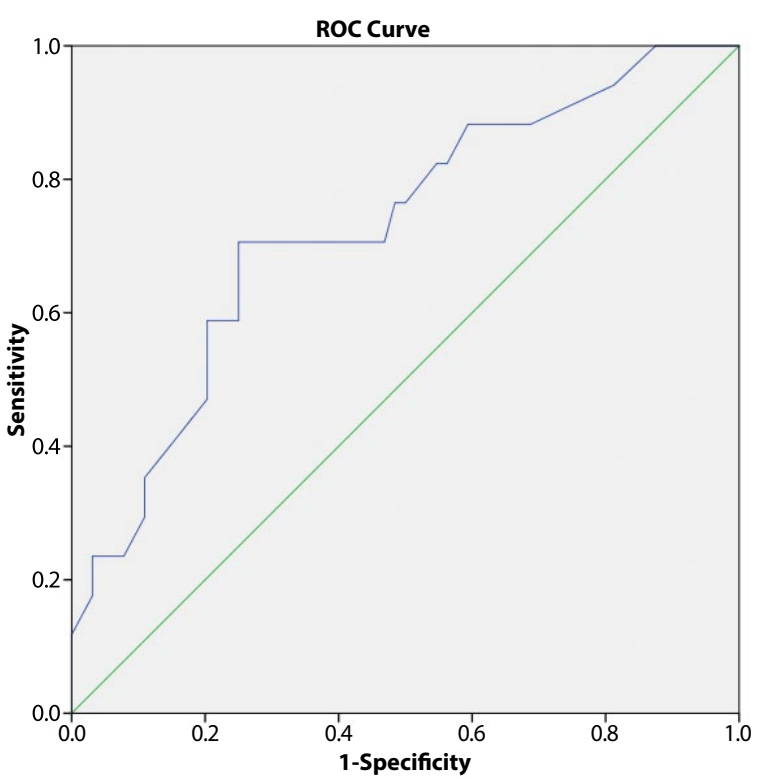

Figure 2. Receiver operating characteristic (ROC) analysis of percentage of subchorionic hematoma surrounding GS in predicting pregnancy outcome. The cut-off value for subchorionic hematoma surrounding GS was $35.5 \%$ while the sensitivity and specificity values were 70 and 75\%, respectively. Area under the ROC curve (AUC): 0.727, sensitivity: $70 \%$, specificity: $75 \%, 95 \% \mathrm{Cl}: 0.590-0.864, \mathrm{p}=0.004$ GS - gestational sac

of negative pregnancy with $70 \%$ sensitivity and $75 \%$ specificity [Area Under the ROC Curve (AUC): 0.727 , sensitivity: 70\%, specificity: $75 \%, 95 \% \mathrm{Cl}: 0.590-0.864, \mathrm{p}=0.004$; Fig. 2]. In the logistic regression analysis to predict the outcome of pregnancy, the effect of NsPP was statistically insignificant. However, if the percentage of $\mathrm{ScH}$ was greater than $35 \%$, the risk of pregnancy loss increased by 4.5 times (Wald: 4.881 , Odss ratio: $4.527,95 \%$ Cl: 1.186-17.281, $\mathrm{p}=0.027)$.

\section{DISCUSSION}

The hematoma diameter is one of the important parameters affecting the clinical outcome in $\mathrm{ScH}$ cases [1].
However, we believe that other parameters other than hematoma diameter have important effects on clinical status. In this study, we aimed to evaluate the effects of NsPP on early complications of pregnancy in patients with abortus imminence and subchorionic hematoma. In our current study, we found that pregnancy was more complicated in $\mathrm{ScH}$ cases with NsPP. We think that $\mathrm{ScH}$ s are often small in the first trimester of pregnancy and complicate pregnancy less, and they appear to occur less frequently in early second trimester pregnancies, but they complicate these pregnancies more. In addition, we think that the hematoma diameter may be larger and pregnancy loss may be higher in pregnant women with NsPP accompanied by vaginal bleeding.

The incidence of vaginal bleeding in our clinic was $11.6 \%$. This result was similar to the literature. In their study, Hasan et al. [13], found that the incidence of vaginal bleeding was 7-25\%. In another study with a larger series of cases, Weiss et al. [14], reported the incidence of vaginal bleeding as $14.2 \%$. In our study the incidence of $\mathrm{SCH}$ in patients presenting with vaginal bleeding was $15.4 \%$. There is a wide range of data for the incidence of $\mathrm{SCH}$ in patients with vaginal bleeding in the literature. In the review of Pearlstone, this rate is stated in a very wide range as $4-22 \%$ [15]. This rate suggests that patients presenting with vaginal bleeding should be examined more carefully for $\mathrm{ScH}$. We believe that our article will be the most accurate information providing data to the literature on this subject.

In our study, we found that the higher the ratio of surrounding hematoma to the gestational sac was, the greater the possibility of pregnancy loss became $[1,7,8,16]$. In their study, Bennett et al. [7], used a subjective evaluation including large, medium, and small values for the ratio of surrounding hematoma to the gestational sac. They reported that the risk of pregnancy loss was three times higher in the group with a greater percentage of hematoma than it was in the other two groups. Bennett et al used a small, medium and large subjective criterion for the diameter of the hematoma. In our study, we reported the size of the hematoma in terms of percentages according to the gestational sac containment.

This numerical value (35.5\%) obtained for the ratio of surrounding hematoma to the gestational sac would be more helpful to obstetricians in predicting the outcome of the treatment and complications that may occur in patients with $\mathrm{ScH}$. In the literature, we have found that many studies on $\mathrm{ScH}$ were mostly evaluated in the first trimester of pregnancy. In these studies, many similar results have been obtained in first trimester $\mathrm{ScH}$ cases related to symptoms, findings, and early and late pregnancy complications [17-19]. However, we could not find any study evaluating the cases of early second trimester $\mathrm{ScH}$. In our clinical experi- 
ence, we have seen a significant number of ScHs in the early second trimester of pregnancy. In addition, we observed that the symptoms, clinical findings, and complications were different in these cases after the first trimester. In our study, vaginal bleeding was the most common symptom in first trimester $\mathrm{ScH}$ cases. There were no other symptom frequently associated with vaginal bleeding. However, in the early second trimester $\mathrm{ScH}$ cases, we found that NsPP was frequently accompanied by vaginal bleeding. In early second trimester $\mathrm{ScH}$ cases, with a percentage of hematoma, the duration of hospitalization was longer. In the group with vaginal bleeding and NsPP, pregnancy loss was statistically higher, but in the logistic regression analysis to predict the outcome of pregnancy, the effect of NsPP was statistically insignificant. Although the effect of NsPP on early pregnancy loss is meaningless in logistic regression analysis; we think that clinicians who are evaluating $\mathrm{ScH}$ s cases should consider this symptom. The ratio of surrounding hematoma to the gestational sac was found to be significantly higher in early second trimester $\mathrm{ScH}$ cases. As this ratio increases, it causes greater separation of the gestational sac from the uterine wall. As a result, inflammatory cell infiltration and inflammatory mediators will occur more around the gestational sac $[20,21]$. These inflammatory mediators result in the formation of smooth muscle-tightening molecules, such as prostaglandin 12 and thromboxane $A 2$ [22, 23]. We think that prostaglandin and thromboxane A2 cause NsPP [24]. In addition, the uterus will be larger than normal for the gestational week in cases where the $\mathrm{ScH}$ is larger $[25,26]$. We think that the stretched visceral peritoneum of the uterus and the inflammatory mediators occurred due to hematoma contribute to the formation of NsPP.

\section{CONCLUSIONS}

$\mathrm{ScH}$ was more common in first trimester pregnancies, which was found to have a smaller diameter and cause fewer complications in pregnancy. Although $\mathrm{ScH}$ was less common in the early second trimester group, it was found to have a larger diameter, so it caused more complications in pregnancy. In addition, in cases of $\mathrm{ScH}$, it should be kept in mind that the hematoma diameter may be larger and pregnancy may be more complicated in cases in which vaginal bleeding is accompanied by NsPP. Furthermore, NsPP cannot be explained for another reason in $\mathrm{ScH}$, should be a stimulant for pregnancy complication. In order to better understand the effect of NsPP on early pregnancy losses, studies with larger case numbers are needed.

\section{REFERENCES}

1. Nagy S, Bush M, Stone J, et al. Clinical significance of subchorionic and retroplacental hematomas detected in the first trimester of pregnancy. Obstet Gynecol. 2003; 102(1): 94-100, doi: 10.1016/s0029-7844(03)00403-4, indexed in Pubmed: 12850613.
2. Truong A, Sayago MM, Kutteh $\mathrm{WH}$, et al. Subchorionic hematomas are increased in early pregnancy in women taking low-dose aspirin. Fertil Steril. 2016; 105(5): 1241-1246, doi: 10.1016/j.fertnstert.2016.01.009, indexed in Pubmed: 26820772.

3. Abu-Yousef MM, Bleicher JJ, Williamson RA, et al. Subchorionic hemorrhage: sonographic diagnosis and clinical significance. AJR Am J Roentgenol. 1987; 149(4): 737-740, doi: 10.2214/ajr.149.4.737, indexed in Pubmed: 3307354.

4. Mandruzzato GP, D'Ottavio G, Rustico MA, et al. The intrauterine hematoma: diagnostic and clinical aspects. J Clin Ultrasound. 1989; 17(7): 503-510, doi: 10.1002/jcu.1870170708, indexed in Pubmed: 2506245.

5. Pedersen JF, Mantoni M. Prevalence and significance of subchorionic hemorrhage in threatened abortion: a sonographic study. AJR Am J Roentgenol. 1990; 154(3): 535-537, doi: 10.2214/ajr.154.3.2106217, indexed in Pubmed: 2106217.

6. Benson $\mathrm{CB}$, Doubilet PM, Cooney MJ, et al. Early singleton pregnancy outcome: effects of maternal age and mode of conception. Radiology. 1997; 203(2): 399-403, doi: 10.1148/radiology.203.2.9114094, indexed in Pubmed: 9114094.

7. Bennett GL, Bromley B, Lieberman E, et al. Subchorionic hemorrhage in first-trimester pregnancies: prediction of pregnancy outcome with sonography. Radiology. 1996; 200(3): 803-806, doi: 10.1148/radiology.200.3.8756935, indexed in Pubmed: 8756935.

8. Heller HT, Asch EA, Durfee SM, et al. Subchorionic Hematoma: Correlation of Grading Techniques With First-Trimester Pregnancy Outcome. J Ultrasound Med. 2018; 37(7): 1725-1732, doi: 10.1002/jum.14524, indexed in Pubmed: 29341210.

9. Tuuli MG, Norman SM, Odibo $A O$, et al. Perinatal outcomes in women with subchorionic hematoma: a systematic review and meta-analysis. Obstet Gynecol. 2011; 117(5): 1205-1212, doi: 10.1097/AOG.0b013e31821568de, indexed in Pubmed: 21508763.

10. Biesiada L, Krekora M, Krasomski G. [Subchorionic hematoma as a risk factor of pregnancy and delivery in women with threatening abortion]. Ginekol Pol. 2010; 81(12): 902-906, indexed in Pubmed: 21395080.

11. Amundsen PA, Evans DW, Rajendran D, et al. Inclusion and exclusion criteria used in non-specific low back pain trials: a review of randomised controlled trials published between 2006 and 2012. BMC Musculoskelet Disord. 2018; 19(1): 113, doi: 10.1186/s12891-018-2034-6, indexed in Pubmed: 29650015.

12. Vas J, Cintado MC, Aranda-Regules JM, et al. Effect of ear acupuncture on pregnancy-related pain in the lower back and posterior pelvic girdle: A multicenter randomized clinical trial. Acta Obstet Gynecol Scand. 2019; 98(10): 1307-1317, doi: 10.1111/aogs.13635, indexed in Pubmed: 31034580 .

13. Hasan $\mathrm{R}$, Baird $\mathrm{DD}$, Herring $\mathrm{AH}$, et al. Patterns and predictors of vaginal bleeding in the first trimester of pregnancy. Ann Epidemiol. 2010; 20(7): 524-531, doi: 10.1016/j.annepidem.2010.02.006, indexed in Pubmed: 20538195.

14. Weiss JL, Malone FD, Vidaver J, et al. FASTER Consortium. Threatened abortion: A risk factor for poor pregnancy outcome, a population-based screening study. Am J Obstet Gynecol. 2004; 190(3): 745-750, doi: 10.1016/j.ajog.2003.09.023, indexed in Pubmed: 15042008.

15. PEARLSTONE M, BAXI L. Subchorionic Hematoma. Obstetrical \& Gynecological Survey. 1993; 48(2): 55-68, doi: 10.1097/00006254199302000-00001.

16. AYDIN O, BOSTAN PA, ÖZGÜR E. Spatial Distribution and Modelling of the Total Fertility Rate in Turkey Using Spatial Data Analysis Techniques. Journal of Geography. 2018: 27-45, doi: 10.26650/jgeog434650.

17. Kurjak A, Schulman $H$, Zudenigo $D$, et al. Subchorionic hematomas in early pregnancy: clinical outcome and blood flow patterns. J Matern Fetal Med. 1996; 5(1): 41-44, doi: 10.1002/(SICI)1520-6661(199601/02) 5:1<41::AID-MFM10>3.0.CO;2-Q, indexed in Pubmed: 8796766.

18. Maso G, D'Ottavio G, De Seta F, et al. First-trimester intrauterine hematoma and outcome of pregnancy. Obstet Gynecol. 2005; 105(2): 339-344, doi: 10.1097/01.AOG.0000152000.71369.bd, indexed in Pubmed: 15684162

19. Johns J, Hyett J, Jauniaux E. Obstetric outcome after threatened miscarriage with and without a hematoma on ultrasound. Obstet Gynecol. 2003; 102(3): 483-487, doi: 10.1016/s0029-7844(03)00580-5, indexed in Pubmed: 12962928.

20. Christiansen $\mathrm{OB}$, Nielsen HS, Kolte AM. Inflammation and miscarriage. Semin Fetal Neonatal Med. 2006; 11(5): 302-308, doi: 10.1016/j. siny.2006.03.001, indexed in Pubmed: 16682265. 
21. Kwak-Kim J,Yang KM, Gilman-Sachs A. Recurrent pregnancy loss: a disease of inflammation and coagulation. J Obstet Gynaecol Res. 2009; 35(4):609-622, doi: 10.1111/j.1447-0756.2009.01079.x, indexed in Pubmed: 19751318.

22. Ricciotti E, FitzGerald GA. Prostaglandins and inflammation. Arterioscler Thromb Vasc Biol. 2011; 31(5): 986-1000, doi: 10.1161/ATVBAHA.110.207449, indexed in Pubmed: 21508345.

23. Jabbour HN, Sales KJ, Smith OP, et al. Prostaglandin receptors are mediators of vascular function in endometrial pathologies. Mol Cell Endocrinol. 2006; 252(1-2): 191-200, doi: 10.1016/j.mce.2006.03.025, indexed in Pubmed: 16701939.
24. Wang $C$, Wang $Z$, Yang $Y$, et al. Pirt contributes to uterine contraction-induced pain in mice. Mol Pain. 2015; 11: 57, doi: 10.1186/s12990-0150054-x, indexed in Pubmed: 26376721.

25. Ganer Herman H, Miremberg H, Dekalo A, et al. Preterm uterine contractions ultimately delivered at term: safe but not out of danger. Eur J Obstet Gynecol Reprod Biol. 2016; 199: 1-4, doi: 10.1016/j.ejogrb.2016.01.019, indexed in Pubmed: 26874238.

26. MeiY, Lin Y.Clinical significance of primary symptoms in women with placental abruption. J Matern Fetal Neonatal Med. 2018; 31(18): 2446-2449, doi: 10.1080/14767058.2017.1344830, indexed in Pubmed: 28629246. 